\title{
REM sleep motor dysfunction in multiple system atrophy: with special emphasis on sleep talk as its early clinical manifestation
}

\author{
N Tachibana, K Kimura, K Kitajima, A Shinde, J Kimura, H Shibasaki
}

\begin{abstract}
Various neurodegenerative diseases involving brainstem structures as one of the main pathological lesions are reported to be associated with REM sleep behaviour disorder. Full blown REM sleep behaviour disorder can be diagnosed clinically, but REM sleep motor dysfunction, a pathophysiological basis of REM sleep behaviour disorder, is difficult to detect without all night polysomnography. Twenty one consecutive patients with multiple system atrophy with no complaints of nocturnal abnormal behaviours were clinically evaluated to determine the presence of sleep related symptoms. All night polysomnography with video monitoring was performed to investigate REM sleep characteristics and patients' behaviours. In $85.7 \%$ (18 of 21 ) of the patients' sleep talk started or increased around or after the clinical onset of the primary diseases. REM sleep without atonia occupied more than $15 \%(16.2 \%-100 \%)$ of the REM sleep time in all but one patient. In $90.5 \%$ (19 of 21) of patients, motor events such as sleep talk and various combinations of craniofacial, orofacial, or limb movements occurred at various frequencies mostly during REM sleep without atonia. In patients with multiple system atrophy, REM sleep motor dysfunction is a common polysomnographic finding which is otherwise overlooked, and sleep talk may be its early clinical manifestation.
\end{abstract}

(F Neurol Neurosurg Psychiatry 1997;63:678-681)

Keywords: multiple system atrophy; sleep talk; REM sleep behaviour disorder

REM sleep without atonia, first described in experimental cats with bilateral lesions in the dorsolateral pontine tegmentum, ${ }^{1}$ was found to be associated with behavioural manifestations, ranging from REM sleep without atonia associated with no behavioural correlates to that with active locomotor disinhibition, depending on the location and size of the lesions. ${ }^{2}$ Experimental cats with active locomotion along with violent behaviours have been considered an animal model for REM sleep behaviour disorder seen in neurological diseases with brainstem lesions. ${ }^{3-5}$ Based on these experimental findings, we hypothesised that REM sleep motor dysfunction might occur even in patients with multiple system atrophy who do not report any nocturnal abnormal behaviours. Furthermore, by utilising all night polysomnography, the only method capable of detecting this kind of REM sleep abnormality that does not fulfill the criteria of REM sleep behaviour disorder, we aimed at elucidating how sleep related clinical symptoms are related to REM sleep motor dysfunction in patients with multiple system atrophy.

\section{Patients and methods}

PATIENTS

We studied 21 consecutive patients admitted to the Department of Neurology, Kyoto University Hospital with the diagnosis of multiple system atrophy (14 patients with sporadic olivopontocerebellar atrophy, six with Shy-Drager syndrome, and one with striatonigral degeneration) between July 1993 and January 1996 (table). All the patients were referred to us either for confirming the diagnosis or for seeking a second opinion, but not because of sleep related complaints. They were seven men and 14 women; mean (SD) age 60.5 (8.9) years, mean duration of disease 2.5 (range 0.5-10) years, and mean severity grade 1.8 (SD 1.1). ${ }^{6}$ The diagnosis was mainly based on the clinical features. None of them had a history of head injury, seizure disorder, psychiatric disorder, or alcohol or other drug misuse. All the patients were free of antiparkinsonian medication or psychoactive drugs for at least two weeks before the polysomnography studies.

METHODS

All patients and family members participated in the interview on their sleep history and sleep habit by using a structured sleep questionnaire. For each patient all night polysomnography recordings were performed using the standard 
Table 1 Sleep related symptoms as disclosed by the structured sleep questionnaire in 21 patients with MSA

\begin{tabular}{|c|c|c|c|c|c|c|}
\hline Patient No & Sleep talk* & Vivid dreamst & Snoring & $\begin{array}{l}\text { Excessive } \\
\text { daytime } \\
\text { sleepiness }\end{array}$ & Insomnia & Nocturia \\
\hline \multicolumn{7}{|l|}{ sOPCA: } \\
\hline 1 & + & - & - & - & - & - \\
\hline 2 & +++ & + & - & - & - & + \\
\hline 3 & +++ & - & - & ++ & ++ & ++ \\
\hline 4 & + & - & - & - & - & - \\
\hline 5 & + & ++ & - & - & - & + \\
\hline 6 & +++ & - & + & - & + & - \\
\hline 7 & +++ & - & - & - & ++ & + \\
\hline 8 & ++ & + & ++ & + & - & - \\
\hline 9 & +++ & + & - & - & - & + \\
\hline 10 & + & + & +++ & ++ & + & - \\
\hline 11 & +++ & ++ & ++ & - & + & + \\
\hline 12 & + & + & ++ & ++ & - & - \\
\hline 13 & ++ & - & +++ & - & + & + \\
\hline 14 & +++ & - & ++ & ++ & - & + \\
\hline \multicolumn{7}{|l|}{ SDS: } \\
\hline 15 & ++ & - & + & - & - & + \\
\hline 16 & + & - & ++ & - & - & + \\
\hline 17 & ++ & - & + & - & - & +++ \\
\hline 18 & + & - & +++ & - & - & - \\
\hline 19 & - & - & + & + & - & + \\
\hline 20 & - & - & - & - & + & + \\
\hline \multicolumn{7}{|l|}{ SND: } \\
\hline 21 & - & - & - & - & - & - \\
\hline
\end{tabular}

${ }^{\star}$ Sleep talk $-=$ no or only rare sleep talk; $+=$ sleep talk present, but infrequent; $++=$ frequent sleep talk, but not every night; $+++=$ sleep talk confirmed by family members every night. + Vivid dreams $-=$ no increase in dreams; $+=$ frequent dreams, but not disturbing; $++=$ frequent and vivid dreams, disturbing.

For the other four items: $+=$ mild; $++=$ moderate; $+++=$ severe

MSA = multiple system atrophy; sOPCA = sporadic olivopontocerebellar atrophy; SDS = Shy-Drager syndrome; SND = striatonigral degeneration.

technique in a secluded hospital room. ${ }^{7} \mathrm{EEG}$, electro-oculography (EOG), EMG (submental, tibialis anterior, and gastrocnemius muscles and other muscles as necessary) (figure), ECG, nasal and oral air flow, and chest and abdominal movements were recorded on a polygraph at a paper speed of $15 \mathrm{~mm} / \mathrm{s}$. Oxygen saturation was simultaneously monitored by pulse oxymetry. The patient was monitored with continuous audiovisual recording, and the ongoing behaviours and movements including sleep talk were charted. Videotapes were reviewed afterwards in reference to the polysomnography records.

Staging of waking and REM sleep was carried out according to standard criteria, ${ }^{7}$ and the modified version by Lapierre and Montplaisir was used for staging REM sleep and REM sleep without atonia as well as for defining phasic EMG events in the chin muscles. ${ }^{8}$ REM sleep without atonia was scored either when tonic EMG activity in the chin was present for $50 \%$ or more of a $20 \mathrm{~s}$ epoch or when the independent $2 \mathrm{~s}$ miniepochs containing the phasic submental EMG events occupied $50 \%$ or more of the 20 s epoch. Other determinants of REM sleep (cyclic temporal distribution, irregular breathing pattern, and appearance of saw toothed waves) and presence of segments with normal REM sleep were also considered to define REM sleep without atonia.

Sleep apnoea was defined as cessation of oral and nasal air flow during sleep, and hypopnoea as amplitude reduction of air flow by more than $50 \%$, both lasting $10 \mathrm{~s}$ or longer. Sleep related oxygen desaturation was determined by dips in $\mathrm{SaO}_{2}$ by more than $4 \%$ from the overnight baseline $\mathrm{SaO}_{2}$.

\section{Results}

SLEEP RELATED SYMPTOMS

The table shows the main sleep related symptoms in the patients. No violent nocturnal behaviours were reported by family members. However, sleep talk started or increased around or after the onset of the primary diseases in $85.7 \%$ (18 of 21 ) of the patients, and in seven of them daily somniloquy was asserted by family members, who, however, did not consider it as a problem. Most of the patients reported sleep fragmentation or unrefreshing sleep and attributed it to frequent nocturia. Six patients identified as having excessive daytime sleepiness or daytime fatigue were either snorers or those with a complaint of sleep fragmentation secondary to nocturia. These sleep related symptoms did not correlate with either duration, severity, or predominant signs of the primary disease.

\section{ALL NIGHT POLYSOMNOGRAPHY STUDY}

REM sleep without atonia occupied more than $15 \%$ (mean (SD) $80.0(27.1) \%$, range 16.2100) of the total REM sleep time in all but one patient. In $90.5 \%$ (19 of 21 ) of patients various movements such as shaking or rotating the head, grimacing, teeth clenching, bruxing, chewing, throwing out or raising an arm, and waving a hand were seen, and in most patients these movements were associated with sleep talk ranging from uttering a word or mumbling to full sentences. These motor events were documented mostly during REM sleep without atonia, and never during non-REM sleep. They rarely occurred during REM sleep associated with chin muscle atonia, but some did appear when there were dense muscle twitches in the limb EMG. The movements of the limbs were not clearly purposeful or highly elaborated. Ambulatory behaviours such as sitting up or getting out of the bed were never seen, and no definite dream enacted behaviours occurred. The frequency of these motor events was variable, and did not correlate with either duration, severity, or predominant signs of the primary disease.

REM/REM sleep without atonia periods maintained their cyclic temporal distribution in $85.7 \%$ (18 of 21 ) of patients, and the mean (SD) number of REM periods was 2.5 (2.1) (range 1 to 5). In most cases REM sleep without atonia was characterised by continuous augmentation in chin muscle activity, often accompanied by phasic EMG discharges in the limbs, but on some occasions, independent but repetitive phasic EMG activations in the chin muscles were seen with or without muscle twitching in the limbs (fig 1).

Although obstructive apnoea and hypopnoea were seen in $28.6 \%(6 / 21)$ of patients, none of the motor events was associated with transient arousals with resumption of breathing.

\section{Discussion}

None of our patients could be clinically diagnosed with REM sleep behaviour disorder according to the diagnostic criteria in the International Classification of Sleep Disorders, ${ }^{9}$ but REM sleep without atonia was 


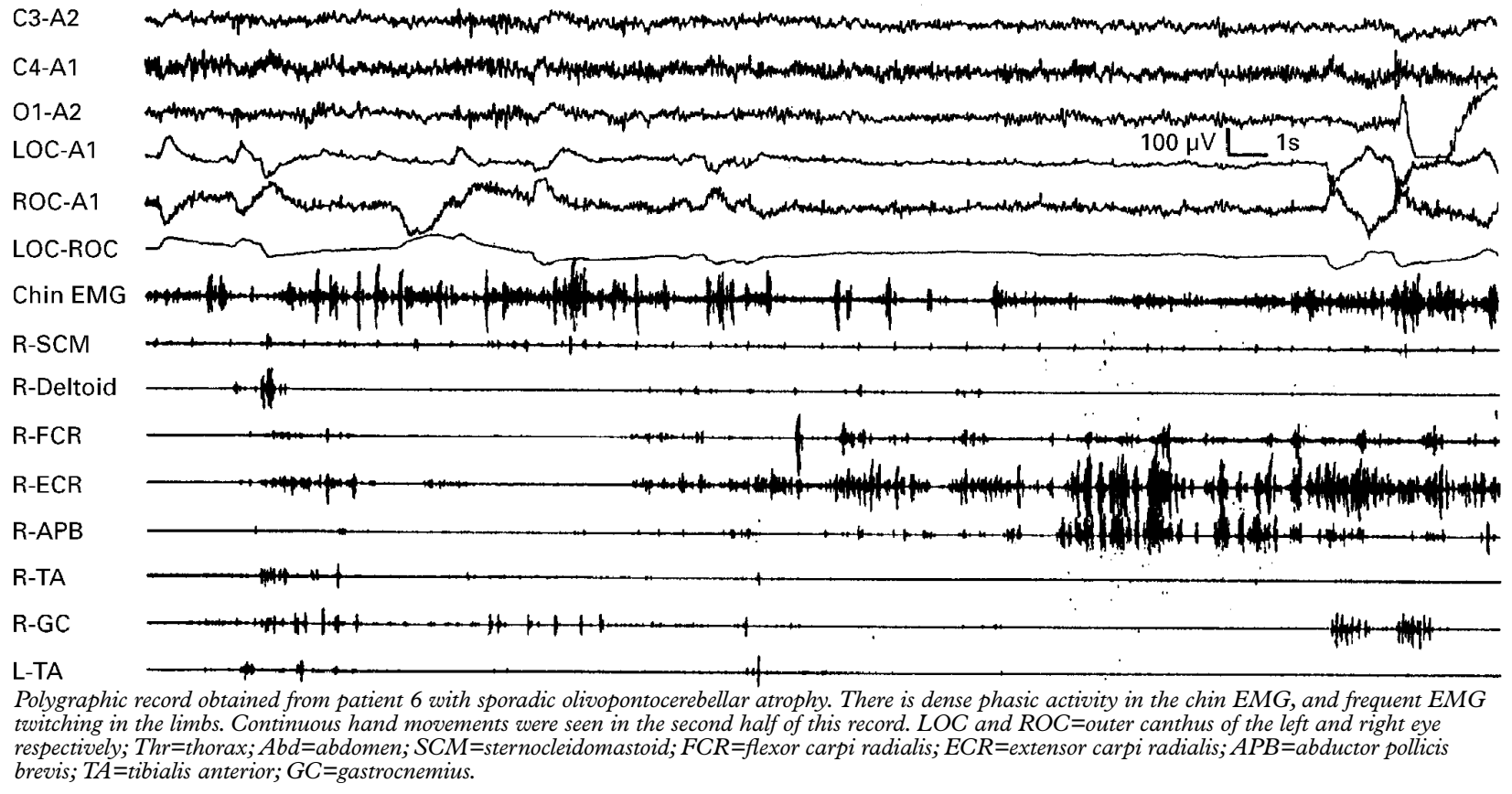

seen in a high percentage of our patients with multiple system atrophy, and compared with the result obtained in normal elderly people, ${ }^{10}$ their percentage of REM sleep without atonia in total REM sleep time was abnormally high. REM sleep behaviour disorder-like phenomena were first anecdotally reported in sporadic olivopontocerebellar atrophy and Shy-Drager syndrome in the early $1980 \mathrm{~s},{ }^{11-13}$ although called by different names. More recently Plazzi et al reported that 35 out of 39 patients with multiple system atrophy had REM sleep behaviour disorder based on polysomnographic recordings. ${ }^{5}$ The mean (SD) duration of the primary disease in their patients was longer (5 (3) years), and $15 \%$ of their patients were referred for prominent sleep related complaints. The findings of Plazzi et al and ours suggest that, even in patients with multiple system atrophy who are not clinically diagnosed with REM sleep behaviour disorder according to the current standard criteria, REM sleep motor dysfunction is commonly present. Although experimental lesion studies in cats suggest that REM sleep behaviour disorder and REM sleep motor dysfunction of various degrees comprise a range of the same pathophysiology, ${ }^{2}$ the mechanism underlying the generation of violent behaviours or minor movements in patients with REM sleep without atonia has not yet been clarified, and the reason why our patients did not present full blown REM sleep behaviour disorder is unknown. A longitudinal study including sleep diaries kept by family members as well as repeated polysomnographic recordings will be required to clarify this issue.

Another interesting clinical finding is that, in half of our patients, sleep talk began or increased in its frequency around or after the onset of the primary disease. There has been no systematic study about the incidence and frequency of sleep talk in the general population. Our patients and their families were ques- tioned only retrospectively, which has a methodological limitation. As sleep talk is noticed much more easily by a bed partner or family members than the patients themselves, it might have been overestimated. In most of our patients, however, sleep talk during REM sleep without atonia was confirmed by the all night polysomnography, and various minor movements of the head, face, neck, and limbs were also simultaneously noted. Pathophysiological studies on sleep talk have been very scarce, but in one monograph, by Arkin, ${ }^{14}$ some of the presented polysomnographic features that had been recorded during sleep talk seem to be consistent with REM sleep without atonia, although the author named it "dissociated sleep stage". The same kind of REM sleep motor dysfunction was reported in sleep talkers. ${ }^{15}$ Therefore, the sleep talk noticed by the families of our patients with multiple system atrophy can be regarded as one of the manifestations of REM sleep motor dysfunction, although the physiology of independent sleep talk seen in otherwise healthy subjects might be various. ${ }^{14}$

MSA has brainstem lesions that interrupt the pontomedullary pathways mediating the REM sleep atonia anatomically and functionally, although REM sleep without atonia is not specific for multiple system atrophy. ${ }^{16}$ In addition, REM sleep behaviour disorder seen in familial fatal insomnia which is characterised by an isolated lesion of the anteroventral and dorsomedial thalamic nuclei suggests that the underlying mechanism of REM sleep without atonia may be heterogeneous. ${ }^{17}$ Neurologists have not been accustomed to systematically inquiring on the presence of sleep related symptoms, but increased or excessive sleep talk should be borne in mind as an early manifestation of REM sleep motor dysfunction in patients with multiple system atrophy. 
We are grateful to Dr I Akiguchi for referring patients under his care, and to Dr H Fukuyama for MRI analysis. This research was supported by grants in aid for scientific research (A) 06404031 and (A)08558083, for International Scientific Research 07044258 , and for Scientific Research on Priority Areas 08279106 from the Japan Ministry of Education, Science, Sports and Culture, an health from the Japan Ministry of Health and Welfare, all for HS.

1 Jouvet M, Delorme F. Locus coeruleus et sommeil paradoxical. Comptes Rendus des Séances de la Société de Biologie (Paris) 1965;159:895-9.

2 Hendricks JC, Morrison AR, Mann GL. Different behaviors during paradoxical sleep without atonia depend on pontine lesion site. Brain Res 1982;239:81-105.

3 Culebras A, Moore JM. Magnetic resonance findings in REM sleep behavior disorder. Neurology 1989;39:1519-23.

4 Mahowald MW, Schenck CH. REM sleep behavior disorder. In: Kryger MH, Roth T, Dement WC, eds. Principals and practice of sleep medicine. Philadelphia: WB Saunders, 1994:575-88.

5 Plazzi G, Corsini R, Provini F, et al. REM sleep behavior disorder in multiple system atrophy. Neurology 1997;48: disorder

6 Hirayama K, Takayanagi T, Nakamura R, et al. Spinocerebellar degeneration in Japan: a nationwide epidemiological and
22 .

7 Rechtschaffen A, Kales A. A manual of standardized terminology, techniques, and scoring system for sleep stages of human subjects. Bethesda, MD: National Institute of Neurological Diseases and Blindness, 1968

8 Lapierre O, Montplaisir J. Polysomnographic features of REM sleep behavior disorder: development of a scoring method. Neurology 1992;42:1371-4.
9 Diagnostic Classification Steering Committee, Thorpy MJ, Chairman. International classification of sleep disorders: diagnostic and coding manual. Rochester, MN: American Sleep Disorders Association, 1990.

10 Tachibana N, Sugita Y, Terashima K, Teshima Y, Shimizu T, Hishikawa Y. Polysomnographic characteristics of the healthy elderly subjects with somnambulism-like behaviors. Biol Psychiatry 1991;30:4-14.

11 Shimizu T, Sugita Y, Iijima S, Teshima Y, Hishikawa Y. Sleep study in patients with spinocerebellar degeneration and related diseases. In: WP Koella, ed. Sleep 1980. Basel: Karger, 1981:435-7.

12 Shimizu T. A polygraphic study of nocturnal sleep in degenerative diseases-a possible mechanism of nocturnal delirium on patients with organic brain conditions. Advances in Neurological Sciences (Shinkei Kenkyu no Shinpo) (Tokyo) 1985;29:154-177 (in Japanese).

13 Quera Salva MA, Guilleminault C. Olivopontocerebellar degeneration, abnormal sleep, and REM sleep without atonia. Neurology 1985;36:576-7.

14 Arkin AM. Sleep talking: psychology and psychophysiology. Hillsdale, New Jersey: Lawlence Erlbaum Associates, 1981.

15 Taniguchi M, Sugita Y, Tachibana N, et al. Sleep talking in REM sleep could be the prodrormal symptom of REM sleep behavior disorder [abstract]. Sleep Research 1995;24A: 242.

16 Tachibana N, Kimura K, Kitajima K, Nagamine T, Kimura J, Shibasaki H. REM sleep without atonia at early stage of sporadic olivopontocerebellar atrophy. F Neurol Sci 1995; 132:28-34.

17 Tinuper P, Montagna P, Medori P, et al. The thalamus participates in the regulation of the sleep-waking cycle. A clinico-pathological study in the fatal familial thalamic degeneration. Electroencephogr Clin Neurophysiol 1989;73: $117-23$. 